\title{
BMJ Open Towards quantifying the reciprocal associations between frequency of cannabis use and alcohol consumption: a cross-lagged analysis from the CONSTANCES cohort
}

\author{
Guillaume Airagnes (DD , ${ }^{1,2}$ Joane Matta (1) ,2 Frédéric Limosin, ${ }^{1,3}$ Nicolas Hoertel, ${ }^{1,3}$ \\ Marcel Goldberg, ${ }^{2}$ Marie Zins, ${ }^{2}$ Cedric Lemogne ${ }^{1,3}$
}

To cite: Airagnes G, Matta J, Limosin F, et al. Towards quantifying the reciprocal associations between frequency of cannabis use and alcohol consumption: a cross-lagged analysis from the CONSTANCES cohort. BMJ Open 2022;12:e052819. doi:10.1136/ bmjopen-2021-052819

- Prepublication history and additional supplemental material for this paper are available online. To view these files, please visit the journal online (http://dx.doi.org/10.1136/ bmjopen-2021-052819).

Received 28 April 2021 Accepted 26 January 2022

Check for updates

(c) Author(s) (or their employer(s)) 2022. Re-use permitted under CC BY-NC. No commercial re-use. See rights and permissions. Published by BMJ.

${ }^{1}$ Department of Psychiatry and Addictology, AP-HP.CentreUniversité de Paris, Paris, Île-deFrance, France

${ }^{2}$ UMS011, INSERM, Villejuif,

France

${ }^{3}$ Université de Paris, INSERM, Institut de Psychiatrie et Neurosciences de Paris (IPNP), UMR_S1266, Paris, France

Correspondence to Dr Guillaume Airagnes; guillaume.airagnes@aphp.fr

\section{ABSTRACT}

Objective Disentangle the temporal relationships between frequency of cannabis use and alcohol consumption. Methods A cross-lagged model providing standardised coefficients (SCs) \pm their standard errors in 13255 men and 13696 women enrolled in 2015 or 2016 in the French population-based 'CONSulTANts des Centres d'Examens de Santé' (CONSTANCES) cohort. Cannabis use was categorised as follows: 'No use during the past 12 months', 'Use during the past 12 months but not in the past month' and 'Use in the past month' for cannabis use at baseline, and No use during the past 12 months, 'Use less than once per month' and 'Use once per month or more' for cannabis use at 1 year of follow-up. Alcohol consumption was measured at baseline and at 1 year of follow-up and three categories were determined: low risk ( $<28$ drinks per week in men; $<14$ drinks per week in women), moderate risk ( $\geq 28$ and $<42$ in men; $\geq 14$ and $<28$ in women) and high risk ( $\geq 42$ in men; $\geq 28$ in women). Analyses were adjusted for age, sex, education, income, tobacco consumption, self-rated health status and depressive symptoms.

Results Both associations from alcohol to cannabis and from cannabis to alcohol were significant $(S C=0.02 \pm 0.01$ with $\mathrm{p}=0.003$ and $\mathrm{SC}=0.06 \pm 0.01$ with $\mathrm{p}<0.001$, respectively). However, the $\mathrm{SC}$ of the association from cannabis to alcohol was three times higher than the opposite association $(p<0.001)$. After stratification for sex, SCs of the association from cannabis to alcohol were more than two times higher than for the opposite association in men, and more than four times higher in women (both $p<0.001$ ).

Conclusions The association between frequency of cannabis use and subsequent alcohol consumption was stronger than the opposite association. This finding encourages considering the risk of increased alcohol consumption among cannabis users.

\section{INTRODUCTION}

Substance use is one of the first leading preventable cause of premature death worldwide. ${ }^{1}$ At a population level, alcohol, tobacco
Strengths and limitations of this study

- The present study used longitudinal data to perform cross-lagged models that allow quantifying and comparing the coefficients of the direct effects of frequency of cannabis use on alcohol consumption with the opposite association.

- Both main predictors/outcomes of interest were measured at both baseline and 1-year follow-up in a large sample from a national population-based cohort.

- The potential confounding effects of sociodemographic and clinical variables were taken into account.

- Since participants from the CONSTANCES cohort are not representative of the general population, our findings may not apply to other settings.

- The observational nature of the data restricts the possibility of drawing confirmed causality.

and other drugs, especially cannabis, which is the most commonly illicit drug used, are among the leading risk factors for morbidity and mortality worldwide. ${ }^{2}$ In addition, co-use of these substances may be associated with increased levels of consumption, dependency and deleterious health consequences. ${ }^{3-6}$ Temporal relationships have been particularly described in the co-use of tobacco and cannabis in one hand, ${ }^{7-10}$ and in the co-use of tobacco and alcohol in the other hand. ${ }^{11} 12$ Although associations between cannabis and alcohol use have been also described, the literature poorly examined whether the consumption of one could increase the consumption of the other over time, besides acute intoxication. ${ }^{13}$

The ongoing policy changes in several countries regarding legalisation or decriminalisation of cannabis use should encourage 
examining the potential detrimental role of cannabis on alcohol consumption, even in occasional cannabis users. ${ }^{61415}$ As a matter of fact, higher levels of alcohol consumption were reported among cannabis users, as well as greater negative social and behavioural consequences of their alcohol use. ${ }^{13-17}$ Regarding the direction of the association, a longitudinal study found a higher incidence of alcohol abuse or dependence over a 3-year follow-up period among subjects with cannabis use compared with those who did not use cannabis at baseline. ${ }^{18}$ This might be interpreted as a harmful consequence of cannabis use on the risk of alcohol use disorder. However, this association might reflect the increased risk of developing another addictive behaviour after the occurrence of a first one, irrespective of the type of the substance that was initially used. In other words, the risk of developing an addictive behaviour when the first one is present is high, but the extent to which the reciprocal association is similar, stronger or smaller remains unknown. However, this knowledge would be helpful for both clinicians and public health policy-makers to improve information and targeted screening.

We took advantage of the longitudinal data from the French population-based CONSTANCES cohort to disentangle the temporal relationships between frequency of cannabis use and alcohol consumption using a crosslagged model. ${ }^{19}$ The cross-lagged model can provide information on the direction of the association between cannabis and alcohol by quantifying the magnitude of the two directions simultaneously while taking into consideration potential confounding factors. We hypothesised a significantly higher magnitude of the association from cannabis to alcohol compared with the opposite association, suggesting that this direction would prevail over the other.

\section{METHODS}

\section{Cohort description}

The French population-based CONSTANCES cohort enrolled volunteers aged 18-69 years at baseline according to a random sampling scheme stratified on age, gender, socioeconomic status and region of France..$^{19} 20$ Among the different procedures conducted with participants, they completed annual self-administered questionnaires on their lifestyle, health, social and personal characteristics.

\section{Patient and public involvement}

Patients or members of the public were not involved in the design of this study, nor in its implementation. Patients and general public will be informed of the results of the study via publication.

\section{Participants}

In the present study, we used data from 47673 participants enrolled in the CONSTANCES cohort in 2015 or 2016 and to whom the questionnaire on alcohol habits had been sent at both baseline and 1 year of follow-up. Among them, 37588 (78.8\%) had no missing data regarding their alcohol, cannabis and tobacco use at baseline. Complete data for substance use were available at follow-up for 27696 participants (73.7\%). Cannabis use was illicit in France when the data were collected. Among the non-responders at follow-up, 9276 (87.3\%) did not respond to the entire questionnaire whereas $1361(12.7 \%)$ did not respond to the questions related to substance use. Finally, since we were interested in examining the changes in alcohol consumption among persons likely to consume alcohol, the 745 participants who reported to having never consumed alcohol in their life were excluded. Thus, a total of 26951 participants were included in the present study. A description of their characteristics before and after attrition is presented in online supplemental table 1.

\section{Measurements}

Cannabis use at baseline and at follow-up

The questionnaire at baseline included three binary questions that were used to determine cannabis consumption: (1) 'Have you ever used cannabis (hashish, marijuana, weed, joint, pot)?', (2) 'Have you used cannabis in the past 12 months?' and (3) 'Have you used cannabis in the past thirty days?'. From this information, a categorical variable was computed as follows: 'No use during the past 12 months', 'Use during the past 12 months but not in the past month' and 'Use in the past month'.

The questionnaire at 1 year of follow-up included the following binary question: 'Over the past 12 months, have you used cannabis (hashish, marijuana, weed, joint, pot)?'. Those who answered 'yes' were asked to further provide information on how often they had used cannabis with the following options: 'Less than once per month', 'Once to twice per month', 'At least once per week', 'Every day, or nearly'. From this information, a categorical variable was computed as follows: No use during the past 12 months, 'Use less than once per month' and 'Use once per month or more'. Since measurements of cannabis frequency of use slightly differ at baseline and at follow-up, sensitivity analyses were conducted. In these analyses, we used the answers to the following question: 'How many times have you used it over the past 12 months?', that was asked among those who reported a cannabis use in the past 12 months prior to baseline. To compensate for a potential underestimation of the number of monthly users at baseline, we also considered as users of once per month or more those who did not report a cannabis use in the past 30 days but who reported $\geq 12$ times of cannabis use in the past 12 months. Conversely, to attenuate a potential overestimation of the number of monthly users at baseline, we considered as users of less than once per month those who reported cannabis use in the past 30 days but who reported $<12$ times of cannabis use in the past 12 months. 
Alcohol use at baseline and at follow-up

At both baseline and 1 year of follow-up, participants reported their daily alcohol consumption during the past week in number of drinks, considering that a drink includes $10 \mathrm{~g}$ of alcohol according to the standard in France. $^{21}$ Precisely, participants reported their mean number of drinks per day from 'Monday to Thursday', and their number of drinks on 'Friday', 'Saturday' and 'Sunday'. These numbers were added after having multiplied by 4 the sum corresponding to the line from Monday to Thursday in order to compute the number of drinks per week. Thus, three risk categories according to the level of alcohol consumption were built based on the WHO guidelines: alcohol consumption at low $(<28$ drinks per week in men; $<14$ drinks per week in women), moderate ( $\geq 28$ and $<42$ in men; $\geq 14$ and $<28$ in women) or high risk ( $\geq 42$ in men; $\geq 28$ in women) ${ }^{22}$

\section{Covariables at baseline}

The following sociodemographic variables were obtained from the baseline questionnaire: age, sex, education coded in five levels based on the 2011 International Standard Classification of Education, ${ }^{23}$ household income (ie, <€2100; €2100-2800; €2800-4200; >€4200/month) and marital status (ie, single or living with a partner). Education and household income were considered as ordinal representation of underlying sets of continuous units (ie, years of education and amount of money in euros per month); thus, these two variables were introduced as continuous variables in the statistical analyses. ${ }^{24}$ Daily tobacco consumption was computed as a continuous variable in cigarettes per day from the cumulative number of tobacco consumption per day whatever the product used (eg, common cigarette, cigar and pipe). Self-rated health status was assessed as a continuous variable by answering the following question: 'How do you judge the state of your general health compared to a person of your surrounding of the same age' on an 8-point like scale (1=very good, 8 =very poor). ${ }^{25}$ Depressive symptoms were assessed as a continuous variable using the total score at the Center of Epidemiologic Studies Depression scale, ranging from 0 to $60 .{ }^{2627}$

\section{Statistical analyses}

The association between the two main outcomes and predictors of interest (ie, cannabis and alcohol at both baseline and follow-up) was tested using a cross-lagged model. The cross-lagged model is a structural equation model that considers all variables together, along with the covariables. In a cross-lagged model, results are presented as standardised coefficients which correspond to the coefficients of the regressions that are standardised to allow comparing the relative effects of two variables on each other. ${ }^{28}$ Conditional on their standard errors, the comparison of these standardised coefficients can determine which variable has a stronger effect on the other, thus informing on which direction of the association predominates over the other. ${ }^{29}$ Cross-lagged analyses were conducted with SAS Proc Calis (SAS, V.9.4). As described in this SAS procedure, standardised coefficients are obtained by introducing standardised variables in the models, that is, variables that have been centred and divided by their SD whether they consist of ordered categories or whether they are binary or continuous. ${ }^{30}$

Frequency of cannabis use and alcohol consumption at both baseline and 1 year of follow-up were included in the model as ordinal variables, that is, ordinal representations of underlying sets of continuous units. ${ }^{24}$ Furthermore, prior findings suggested dose-dependent associations of these variables with detrimental outcomes. ${ }^{31}{ }^{32}$ Residuals were allowed to correlate. Standardised coefficients were obtained in order to compare both direct effects (ie, from alcohol to cannabis and from cannabis to alcohol). The model was adjusted for the following covariables at baseline: age, sex, education, household income, marital status, depressive symptoms and self-rated health status. Covariables were allowed to correlate. In the path model, because we sought to examine simultaneously all path coefficients, no paths in any of the models were fixed to zero. Therefore, goodness-of-fit measures are not relevant in evaluating these models since they do not inform on the 'correctness' of the models but rather provide only a summary of how well the observed correlations match the model when several paths are fixed at zero. ${ }^{33} 34$ Some fit indices are however reported in the results order to show the goodness of fit of the model using the following cut-offs: Standardized Root Mean squared Residual $(\mathrm{SRMR})<0.08$, Goodness of Fit Index $(\mathrm{GFI})>0.95$, Root Mean Square Error of Approximation (RMSEA) $<0.05$ and Normed Fit Index $(\mathrm{NFI})>0.95$. Since substance use patterns could differ according to sex, ${ }^{35}$ the analysis of the cross-lagged model has been repeated by stratifying for sex.

To test the robustness of our results, we reproduced the analyses by introducing alcohol and cannabis use as binary variables $(<14$ drinks per week in women and $<28$ drinks per week in men versus $\geq 14$ drinks per week in women and $\geq 28$ drinks per week in men for alcohol use, and no consumption in past 12 months versus consumption in the past 12 months for cannabis use).

Included subjects had complete data regarding dependent variables and tobacco consumption. We had missing data for the other variables, except for age and sex (mean percentage of missing data: $5.0 \%$, going from $1.0 \%$ to $13.5 \%)$. Imputation by stochastic regressions was used to deal with these missing data. ${ }^{24}$ Statistical significance was evaluated with an alpha a priori set at 0.05 .

\section{RESULTS}

A total of 13255 (49.2\%) men and 13696 (50.8\%) women were included, with a mean age of $47.5(\mathrm{SD}=13.4)$. Their characteristics at baseline are presented in table 1. At follow-up, $973(3.6 \%)$ and $629(2.3 \%)$ of the participants used cannabis less than once a month and once a month or more, respectively. At follow-up, 2272 (8.4\%) and 
Table 1 Characteristics of included participants at baseline

\begin{tabular}{|c|c|c|c|c|c|c|}
\hline \multirow[b]{2}{*}{ Categorical variables } & \multicolumn{2}{|c|}{$\begin{array}{l}\text { All participants } \\
\mathrm{N}=26951\end{array}$} & \multicolumn{2}{|l|}{$\begin{array}{l}\text { Men } \\
\mathrm{N}=13255\end{array}$} & \multicolumn{2}{|c|}{$\begin{array}{l}\text { Women } \\
\mathrm{N}=13696\end{array}$} \\
\hline & $\mathbf{N}$ & $\%$ & $\mathbf{N}$ & $\%$ & $\mathbf{N}$ & $\%$ \\
\hline \multicolumn{7}{|l|}{ Education level* } \\
\hline Early childhood education and primary education & 358 & 1.3 & 200 & 1.5 & 158 & 1.2 \\
\hline Lower secondary education & 1151 & 4.3 & 600 & 4.5 & 551 & 4.0 \\
\hline $\begin{array}{l}\text { Upper secondary education and postsecondary } \\
\text { non-tertiary education }\end{array}$ & 8267 & 30.7 & 4561 & 34.4 & 3706 & 27.1 \\
\hline $\begin{array}{l}\text { Short-cycle tertiary education and Bachelor's or } \\
\text { equivalent level }\end{array}$ & 9873 & 36.6 & 4059 & 30.6 & 5814 & 42.5 \\
\hline $\begin{array}{l}\text { Master's or equivalent level and Doctoral or } \\
\text { equivalent level }\end{array}$ & 7302 & 27.1 & 3835 & 28.9 & 3467 & 25.3 \\
\hline \multicolumn{7}{|l|}{ Household income (in euros per month) } \\
\hline Less than 2100 & 4423 & 16.4 & 1871 & 14.1 & 2552 & 18.6 \\
\hline From 2100 to 2800 & 4084 & 15.2 & 1859 & 14.0 & 2225 & 16.2 \\
\hline From 2800 to 4200 & 9140 & 33.9 & 4605 & 34.7 & 4535 & 33.1 \\
\hline More than 4200 & 9304 & 34.5 & 4920 & 37.1 & 4384 & 32.0 \\
\hline \multicolumn{7}{|l|}{ Marital status } \\
\hline Living with a partner & 19744 & 73.3 & 10138 & 76.5 & 9606 & 70.1 \\
\hline Single & 7207 & 26.7 & 3117 & 23.5 & 4090 & 29.9 \\
\hline \multicolumn{7}{|l|}{ Alcohol use risk categories $\dagger$} \\
\hline Low & 24631 & 91.4 & 12295 & 92.8 & 12336 & 90.1 \\
\hline Moderate & 1797 & 6.7 & 643 & 4.9 & 1154 & 8.4 \\
\hline High & 523 & 1.9 & 317 & 2.4 & 206 & 1.5 \\
\hline \multicolumn{7}{|l|}{ Cannabis frequency of use } \\
\hline Not in the past 12 months & 24875 & 92.3 & 12007 & 90.6 & 12868 & 94.0 \\
\hline In the past 12 months but not in the past month & 1088 & 4.0 & 619 & 4.7 & 469 & 3.4 \\
\hline In the past month & 988 & 3.7 & 629 & 4.7 & 359 & 2.6 \\
\hline Continuous variables & Mean & SD & Mean & SD & Mean & SD \\
\hline Age & 47.5 & 13.4 & 48.5 & 13.3 & 46.6 & 13.4 \\
\hline Number of cigarettes per day & 1.4 & 4.4 & 1.6 & 4.9 & 1.3 & 3.9 \\
\hline CESD total score & 14.1 & 5.3 & 13.1 & 4.9 & 15.0 & 5.4 \\
\hline Self-rated health status $\ddagger$ & 2.7 & 1.3 & 2.7 & 1.3 & 2.7 & 1.3 \\
\hline
\end{tabular}

*According to the 2011 International Standard Classification of Education (ISCED) classification.

†According to the $2000 \mathrm{WHO}$ guidelines as follows: low risk ( $\leq 28$ drinks per week in men; $\leq 14$ drinks per week in women), moderate risk ( $>28$ and $\leq 42$ in men; $>14$ and $\leq 28$ in women) and high risk (>42 in men; $>28$ in women).

‡From 1='Very good general health' to $8=$ =Very poor general health'.

CESD, Center of Epidemiologic Studies Depression Scale.

$818(3.0 \%)$ of the participants consumed alcohol with moderate and high risks, respectively.

The results of the cross-lagged analysis are presented in figure 1. This model showed a good fit: $S R M R=0.020$, GFI $=1.000$, RMSEA $=0.033(90 \%$ CI 0.030 to 0.035$)$ and $\mathrm{NFI}=0.987$. Both associations from alcohol to cannabis and from cannabis to alcohol were significant: standardised coefficient $=0.02 \pm 0.01$ with $\mathrm{p}=0.003$ and standardised coefficient $=0.06 \pm 0.01$ with $\mathrm{p}<0.001$, respectively. However, the standardised coefficient of the association from cannabis to alcohol was three times higher than the opposite association $(\mathrm{Z}=-4.65 ; \mathrm{p}<0.001)$. The standardised coefficients for all the covariables are presented in online supplemental table 2.

The results of the cross-lagged analysis after stratification for sex are presented in figure 2. These models showed good fits: SRMR $=0.020$, GFI $=1.000$, RMSEA $=0.033$ $(90 \%$ CI 0.030 to 0.035$)$ and NFI $=0.987$; SRMR $=0.020$, GFI $=1.000$, RMSEA $=0.033(90 \%$ CI 0.030 to 0.035$)$ and $\mathrm{NFI}=0.987$, in men and women, respectively. In men, the direct effect from alcohol to cannabis was significant (standardised coefficient $=0.02 \pm 0.01$ with $\mathrm{p}=0.047$ ). The 




Figure 1 Cross-lagged associations between frequency of cannabis use and alcohol consumption in 26951 participants, enrolled in 2015 or 2016 in the CONSTANCES cohort. Results on unidirectional arrows are standardised coefficients \pm their standard errors. Results on bidirectional arrows are residual correlations. The follow-up period was 1 year.

direct effect from cannabis to alcohol was also significant (standardised coefficient $=0.05 \pm 0.01$ with $\mathrm{p}<0.001$ ). However, the standardised coefficient of the association from cannabis to alcohol was more than two times higher than the opposite association $(\mathrm{Z}=-2.44$ with $\mathrm{p}=0.010)$. In women, the direct effect from alcohol to cannabis was significant (standardised coefficient $=0.02 \pm 0.01$ with $\mathrm{p}=0.043$ ). The direct effect from cannabis to alcohol was

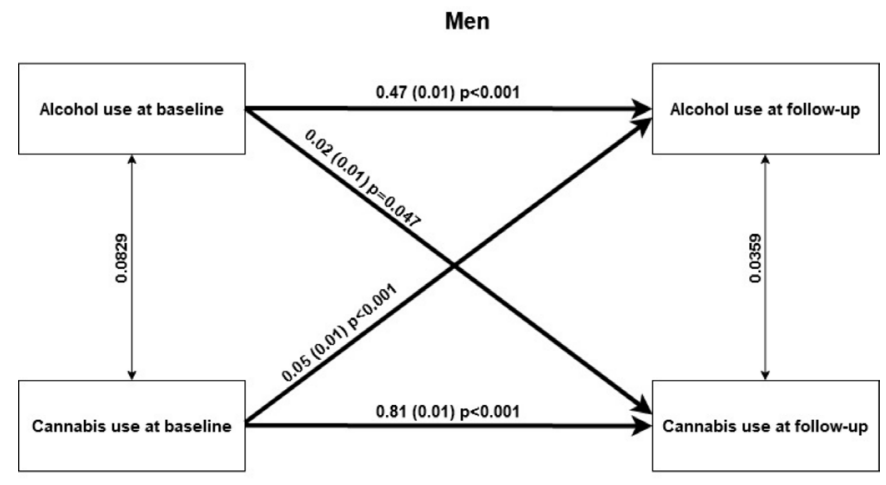

Women

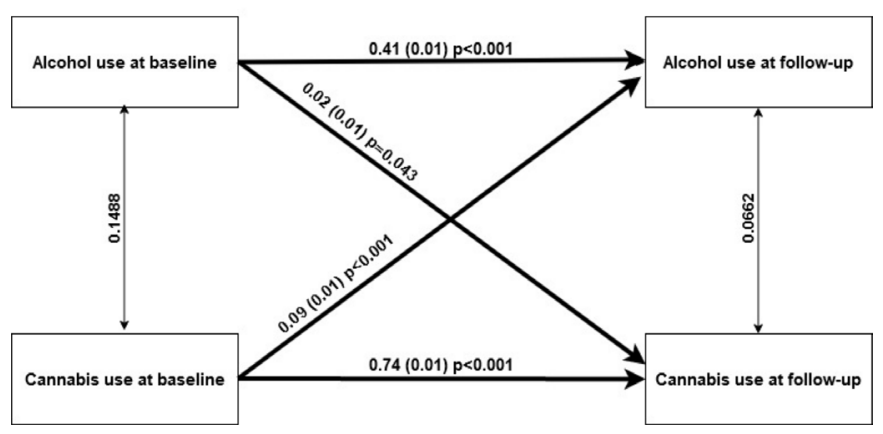

Figure 2 Cross-lagged associations between frequency of cannabis use and alcohol consumption in 13255 men and 13 696 women, enrolled in 2015 or 2016 in the CONSTANCES cohort. Results on unidirectional arrows are standardised coefficients \pm their standard errors. Results on bidirectional arrows are residual correlations. The follow-up period was 1 year. also significant (standardised coefficient $=0.09 \pm 0.01$ with $\mathrm{p}<0.001)$. However, the standardised coefficient from cannabis to alcohol was more than four times higher than the opposite association $(\mathrm{Z}=-5.81 \mathrm{p}<0.0001)$.

In sensitivity analyses, introducing cannabis and alcohol as binary outcomes rather than categorical ones lead similar results, that is, significant associations from alcohol to cannabis and from cannabis to alcohol use, with a significantly higher effect size for the association from cannabis to alcohol than for the opposite association (online supplemental figure 1). Finally, overestimating or underestimating the frequency of cannabis use lead to similar results (data not shown).

\section{DISCUSSION}

Our aim was to disentangle the temporal relationships between frequency of cannabis use and alcohol consumption in a large prospective cohort. In a cross-lagged model, we found that the magnitude of the association from cannabis to alcohol was significantly higher than the opposite (three times higher in the main analysis). After stratification for sex, the higher magnitude of the associations from cannabis to alcohol than the opposite association persisted, with a stronger association in women. All these analyses were adjusted for sociodemographic factors, overall health status and depressive symptoms.

This study has some strengths. Its longitudinal design as well as the use of a cross-lagged model allows quantifying and comparing the coefficients of the direct effects of frequency of cannabis use on alcohol consumption with the opposite association. Both main predictors/outcomes of interest were measured at both baseline and 1-year follow-up in a national population-based cohort having a large sample. In addition, sociodemographic and clinical variables were included in our model to control for potential confounding effects.

This study has also some limitations. First, the CONSTANCES cohort is not representative of the French general population. Thus, our findings may not apply to the same extent to other settings. In particular, participants were aged from 18 to 69 years at inclusion, thus limiting our interpretations to this subgroup. Moreover, all the participants to the CONSTANCES cohort were affiliated to the French General Social Security Regime; thus, certain professional categories could not be represented since they are affiliated to other social regimes, such as independent workers, farmers and craftsmen. Furthermore, the voluntary nature of participation in an epidemiologic cohort leads to selection biases. As it is usually found, there is an under-representation of people with a poor health status in the CONSTANCES cohort (eg, low self-rated health, severe depressive symptoms and very high levels of substance use) ${ }^{20}$ Thus, our findings might not apply to people whose health is the most impaired. Second, there was a $28.3 \%$ attrition rate at follow-up. Although one may wonder whether betweensubstance difference in response rate at follow-up may 
have biased our results toward one association (eg, differential response rate due to the illicit nature of cannabis in France), it is noteworthy that there was a similar rate of non-responders who did not fill specifically their alcohol and cannabis consumption at follow-up, that is, $358(3.37 \%)$ and $365(3.43 \%)$, respectively. Thus, such attrition might have increased a selection bias toward healthier participants but it is unlikely that such bias could act differently on the two substances. Third, the observational nature of the data restricts the possibility of drawing confirmed causality. Fourth, although the results were adjusted for several confounders, some other shared vulnerability factors to both cannabis and alcohol use, such as personality, were not taken into account. These unmeasured confounders may explain to some extent the bidirectional relationship but are unlikely to account for our main result, showing that one direction may substantially prevail on the other.

We found the well-known reciprocal associations between cannabis and alcohol use, which are consistent with the increased likelihood of using a second substance when an individual is already using one, partly because of shared individual and environmental vulnerability factors. ${ }^{36-38}$ Importantly, and in accordance with our a priori hypothesis, we also found that the association between frequency of cannabis use and subsequent alcohol consumption was stronger than the opposite association. This finding is in agreement with the role of the endocannabinoid system on both cannabis and alcohol reinforcement $t^{13} 15$ and with a potential buffering role of cannabis cues on subsequent alcohol cues. ${ }^{39}$ This finding is also in line with prior studies regarding the increased risk of alcohol use disorder according to cannabis use. ${ }^{18}$ Furthermore, the association between frequency of cannabis use and subsequent alcohol consumption was stronger in women. Women could be particularly prone to experience more negative consequences of cannabis use when they also use alcohol. ${ }^{13}$ Men have usually a higher level of alcohol consumption compared with women, ${ }^{40}$ which may reduce the range of variation in consumption in response to other behaviours. This potential novel finding should be further examined in future studies focusing on gender differences.

At an individual level, our findings encourage developing combined treatments for both substances, especially since a treatment focused on cannabis use has shown benefits on alcohol use as well among patients having co-occurring disorders for both alcohol and cannabis. ${ }^{41}$ At a population level, it is noteworthy that the present study deals with very frequently used substances. Indeed, cannabis use is widespread, and even low frequencies of use were associated with subsequent increased alcohol consumption in the present study. Therefore, public health campaigns promoting cannabis cessation should include the potential benefits of such cessation on alcohol consumption. Further studies should focus not only on the level of alcohol consumption but also on the role of the frequency of cannabis co-use on alcohol-related harms. In conclusion, the present study encourages having an increased vigilance regarding the risk of increased alcohol consumption among cannabis users.

Contributors GA: conceptualisation, methodology, software, writing - original draft. JM: methodology, software, writing - review and editing, validation. FL: writing - review and editing, project administration, supervision, validation. $\mathrm{NH}$ : writing - review and editing, visualisation, validation. MG: writing - review and editing, project administration, data curation, resources, investigation, validation. MZ: writing - review and editing, project administration, data curation, resources, investigation, supervision, validation. CL: methodology, writing - review and editing, visualisation, supervision, validation. GA confirms that he had full access to all the data in the study and had final responsibility for the decision to submit for publication.

Funding There are no funders to report for this specific submission. The CONSTANCES cohort is supported by the Caisse Nationale d'Assurance MaladieCNAM. CONSTANCES is accredited as a "National Infrastructure for Biology and health" by the governmental Investissements d'avenir program and was funded by the Agence nationale de la recherche (ANR-11-INBS-0002 Grant). CONSTANCES also receives funding from L'Oréal, MSD, AstraZeneca and Lundbeck managed by INSERM-Transfert. None of the authors are salaried by the funders of the CONSTANCES cohort. The funders did not have any role in the study design, data collection and analysis, decision to publish, or preparation of the manuscript.

Competing interests GA has received speakers and/or consulting fees from Pfizer, Lundbeck, Zentiva and Pierre Fabre, outside the submitted work. FL has received speaker and/or consulting fees from Janssen-Cilag, Euthérapie-Servier, and Lundbeck, outside the submitted work. CL has received speakers and/or consulting fees from Janssen-Cilag, Lundbeck, Otsuka Pharmaceutical, and Boehringer Ingelheim, outside the submitted work.

Patient consent for publication Consent obtained directly from patient(s)

Ethics approval This study involves human participants and was approved by the CONSTANCES cohort has obtained the authorisation of the National Data Protection Authority (Commission Nationale de l'Informatique et des Libertés, no. 910486) and was approved by the Institutional Review Board of the National Institute for Medical Research - INSERM (no. 01-011). Participants gave informed consent to participate in the study before taking part.

Provenance and peer review Not commissioned; externally peer reviewed.

Data availability statement Data are available upon reasonable request. Personal health data underlying the findings of our study are not publicly available due to legal reasons related to data privacy protection. However, the data are available upon request to all interested researchers after authorisation of the French "Commission nationale de l'informatique et des libertés". The CONSTANCES email address is contact@constances.fr.

Supplemental material This content has been supplied by the author(s). It has not been vetted by BMJ Publishing Group Limited (BMJ) and may not have been peer-reviewed. Any opinions or recommendations discussed are solely those of the author(s) and are not endorsed by BMJ. BMJ disclaims all liability and responsibility arising from any reliance placed on the content. Where the content includes any translated material, BMJ does not warrant the accuracy and reliability of the translations (including but not limited to local regulations, clinical guidelines, terminology, drug names and drug dosages), and is not responsible for any error and/or omissions arising from translation and adaptation or otherwise.

Open access This is an open access article distributed in accordance with the Creative Commons Attribution Non Commercial (CC BY-NC 4.0) license, which permits others to distribute, remix, adapt, build upon this work non-commercially, and license their derivative works on different terms, provided the original work is properly cited, appropriate credit is given, any changes made indicated, and the use is non-commercial. See: http://creativecommons.org/licenses/by-nc/4.0/.

\section{ORCID iDs}

Guillaume Airagnes http://orcid.org/0000-0002-3494-5083

Joane Matta http://orcid.org/0000-0002-9123-6726 


\section{REFERENCES}

1 WHO. Global health risks: mortality and burden of disease attributable to selected major risks. World Health Organization, 2009.

2 Peacock A, Leung J, Larney S, et al. Global statistics on alcohol, tobacco and illicit drug use: 2017 status report. Addiction 2018;113:1905-26.

3 Verplaetse TL, McKee SA. An overview of alcohol and tobacco/ nicotine interactions in the human laboratory. Am J Drug Alcohol Abuse 2017;43:186-96.

4 Hayaki J, Anderson BJ, Stein MD. Dual cannabis and alcohol use disorders in young adults: problems magnified. Subst Abus 2016;37:579-83.

5 Linden-Carmichael AN, Mallett KA, Sell N, et al. Are Co-users of alcohol and marijuana more willing to experience consequences from drinking? A longitudinal examination among first-year college students. Alcohol Clin Exp Res 2019;43:1567-74.

6 Goodwin RD. Impact of cannabis use on nicotine and tobacco use outcomes. Nicotine Tob Res 2020;22:1257-9.

7 Agrawal A, Budney AJ, Lynskey MT. The co-occurring use and misuse of cannabis and tobacco: a review. Addiction 2012;107:1221-33.

8 Rabin RA, George TP. A review of co-morbid tobacco and cannabis use disorders: possible mechanisms to explain high rates of co-use. Am J Addict 2015;24:105-16.

9 McClure EA, Rabin RA, Lee DC, et al. Treatment implications associated with cannabis and tobacco Co-Use. Curr Addict Rep 2020;7:533-44.

10 Walsh $\mathrm{H}$, McNeill A, Purssell E, et al. A systematic review and Bayesian meta-analysis of interventions which target or assess co-use of tobacco and cannabis in single- or multi-substance interventions. Addiction 2020;115:1800-14.

11 Beard E, West R, Michie S, et al. Association between smoking and alcohol-related behaviours: a time-series analysis of population trends in England. Addiction 2017;112:1832-41.

12 Piasecki TM, Jahng S, Wood PK, et al. The subjective effects of alcohol-tobacco co-use: an ecological momentary assessment investigation. J Abnorm Psychol 2011;120:557-71.

13 Yurasek AM, Aston ER, Metrik J. Co-use of alcohol and cannabis: a review. Curr Addict Rep 2017;4:184-93.

14 Spithoff S, Emerson B, Spithoff A. Cannabis Legalization: adhering to public health best practice. CMAJ 2015;187:1211-6.

15 Chung T, Harris RA, Cannabis HRA. Cannabis and alcohol: from basic science to public policy. Alcohol Clin Exp Res 2019;43:1829-33.

16 Subbaraman MS, Metrik J, Patterson D, et al. Cannabis use during treatment for alcohol use disorders predicts alcohol treatment outcomes. Addiction 2017;112:685-94.

17 Gunn RL, Norris AL, Sokolovsky A, et al. Marijuana use is associated with alcohol use and consequences across the first 2 years of college. Psychol Addict Behav 2018;32:885-94.

18 Weinberger AH, Platt J, Goodwin RD. Is cannabis use associated with an increased risk of onset and persistence of alcohol use disorders? A three-year prospective study among adults in the United States. Drug Alcohol Depend 2016;161:363-7.

19 Goldberg M, Carton M, Descatha A, et al. CONSTANCES: a general prospective population-based cohort for occupational and environmental epidemiology: cohort profile. Occup Environ Med 2017;74:66-71.

20 Zins M, Goldberg M, CONSTANCES team. The French CONSTANCES population-based cohort: design, inclusion and follow-up. Eur J Epidemiol 2015;30:1317-28.
21 Kalinowski A, Humphreys K. Governmental standard drink definitions and low-risk alcohol consumption guidelines in 37 countries. Addiction 2016;111:1293-8.

22 World Health Organization. International guide for monitoring alcohol consumption and related harm, 2000.

23 Schneider SL. The International Standard Classification of Education 2011. In: Birkelund GE, ed. Class and stratification analysis (comparative social research, volume 30). Emerald Group Publishing Limited, 2013: 365-79.

24 Winship C, Mare RD. Regression models with ordinal variables. Am Sociol Rev 1984;49:512-25.

25 Jylhä M. What is self-rated health and why does it predict mortality? towards a unified conceptual model. Soc Sci Med 2009;69:307-16.

26 Morin AJS, Moullec G, Maïano C, et al. Psychometric properties of the center for epidemiologic studies depression scale (CES-D) in French clinical and nonclinical adults. Rev Epidemiol Sante Publique 2011;59:327-40.

27 Radloff LS. The CES-D scale a self-report depression scale for research in the general population. Appl Psychol Meas 1977;1:385-401.

28 Bentler PM, Speckart G. Attitudes "cause" behaviors: A structural equation analysis. J Pers Soc Psychol 1981;40:226-38.

29 Kearney MW. Cross lagged panel analysis. The SAGE encyclopedia of communication research methods, 2017: 312-4.

30 SAS Institute Inc. SAS/STAT® 13.1 User's Guide. USA: SAS, Cary North Caroline, 2013.

31 Matta J, Hoertel N, Airagnes G, et al. Does substance use explain social differences in terms of depression? findings from the Constances cohort. Compr Psychiatry 2020;102:152203.

32 Airagnes G, Lemogne C, Meneton P, et al. Alcohol, tobacco and cannabis use are associated with job loss at follow-up: findings from the CONSTANCES cohort. PLoS One 2019;14:e0222361.

33 Stage FK, Carter HC, Nora A. Path analysis: an introduction and analysis of a decade of research. J Educ Res 2004;98:5-13.

34 Blanco C, Hoertel N, Wall MM, et al. Toward understanding sex differences in the prevalence of posttraumatic stress disorder: results from the National epidemiologic survey on alcohol and related conditions. J Clin Psychiatry 2018;79.

35 McHugh RK, Votaw VR, Sugarman DE, et al. Sex and gender differences in substance use disorders. Clin Psychol Rev 2018;66:12-23.

36 Roche DJO, Bujarski S, Green R, et al. Alcohol, tobacco, and marijuana consumption is associated with increased odds of same-day substance Co- and tri-use. Drug Alcohol Depend 2019;200:40-9.

37 Olthuis JV, Darredeau C, Barrett SP. Substance use initiation: the role of simultaneous polysubstance use. Drug Alcohol Rev 2013;32:67-71.

38 Sloboda Z, Glantz MD, Tarter RE. Revisiting the concepts of risk and protective factors for understanding the etiology and development of substance use and substance use disorders: implications for prevention. Subst Use Misuse 2012;47:944-62.

39 Müller-Oehring EM, Le Berre A-P, Serventi M, et al. Brain activation to cannabis- and alcohol-related words in alcohol use disorder. Psychiatry Res Neuroimaging 2019;294:111005.

40 Calakos KC, Bhatt S, Foster DW, et al. Mechanisms underlying sex differences in cannabis use. Curr Addict Rep 2017;4:439-53.

41 Dunn HK, Litt MD. Decreased drinking in adults with co-occurring cannabis and alcohol use disorders in a treatment trial for marijuana dependence: evidence of a secondary benefit? Addict Behav 2019;99:106051. 